\title{
Perda de peso, estado de saúde e qualidade de vida durante 2 anos após cirurgia bariátrica
}

\author{
Weight loss, health status and quality of life during 2 years after bariatric surgery
}

\author{
Edina Matilde Linassi Coelho a, Paula Caitano Fontelab, Eliane Roseli Winkelmannc, \\ Maria Simone Vione Schwengber ${ }^{d}$ \\ a Fisioterapeuta. Mestre em Educação nas Ciências pela Universidade Regional do Noroeste do Estado do Rio Grande do Sul (UNIJUÍ). \\ Docente do Departamento de Ciências da Vida da UNIJUÍ, ljuí, RS, Brasil. \\ b Fisioterapeuta. Mestranda do Programa de Pós-Graduação em Ciências Pneumológicas da Universidade Federal do Rio Grande do Sul (UFRGS), Porto Alegre, RS, Brasil. \\ c Fisioterapeuta. Doutora em Ciências da Saúde pela UFRGS. Docente do Programa de Pós-Graduação Stricto Sensu Mestrado em Atenção Integral à Saúde - PPGAIS \\ e do Departamento de Ciências da Vida da UNIJUí, ljuí, RS, Brasil. \\ Educadora física. Doutora em Educação pela UFRGS. Docente do Programa de Pós-Graduação Stricto Sensu em Educação nas Ciências e do \\ Departamento de Humanidades e Educação da UNIJUí, Ijuí, RS, Brasil.
}

RESUMO

Introdução: A cirurgia bariátrica é cada vez mais considerada como alternativa para o tratamento da obesidade extrema.

Objetivo: Descrever a evolução do peso, condições de saúde e qualidade de vida durante 2 anos após a cirurgia bariátrica.

Materiais e Métodos: Estudo de coorte retrospectiva realizada com indivíduos, idade entre 15 a 65 anos, que realizaram qualquer tipo de procedimento cirúrgico bariátrico no período de Janeiro de 2010 a Julho de 2011, em um Centro Terapêutico da Obesidade. Mudanças antropométricas, bioquímicas e da qualidade de vida, condições coexistentes e o resultado final da intervenção cirúrgica pela pontuação Bariatric Analysis and Reporting Outcome System (BAROS) foram avaliadas até o segundo ano após a cirurgia.

Resultados: Foram avaliados 70 indivíduos, $74,3 \%$ do sexo feminino, com 37,2 $\pm 10,9$ anos. Notou-se que o índice de massa corporal (IMC) pré-operatório foi de 45,5 kg/m². Após 2 anos da cirurgia bariátrica, a média percentual de perda de peso foi de 37,3\% (IC95\%: 34,9-39,7\%). Observou-se também remissão de 100\% dos casos de diabetes mellitus, hipertensão arterial sistêmica e apneia noturna; $97,4 \%$ da hipoventilação; 87,5\% da estenose venosa; $75 \%$ da dislipidemia e $61,7 \%$ da osteoartrite. Em relação aos exames bioquímicos, notou-se redução dos valores de colesterol total e LDL neste período $(p=0,042 ; p=0,021$, respectivamente). A qualidade de vida, segundo o questionário específico de BAROS, melhorou significativamente no primeiro ano após a cirurgia, comparado aos 6 meses iniciais, nos domínios estado físico, convívio social e capacidade para o trabalho $(p=0,008 ; p=0,001$; $p=0,035$; respectivamente), e no segundo ano no domínio desempenho sexual $(p=0,008)$. Na análise do resultado final da intervenção cirúrgica, prevaleceu a categoria "bom" em todos os momentos avaliados.

Conclusão: Após um ano da realização da cirurgia bariátrica, observa-se redução do peso, melhora na saúde cardiometabólica e geral e na qualidade de vida dos indivíduos.

Palavras-chaves: obesidade; qualidade de vida; cirurgia bariátrica; estado de saúde; Brasil.

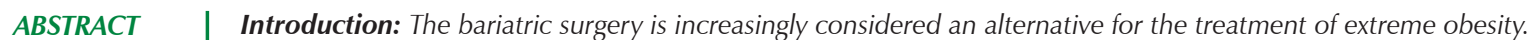

Objective: To describe the weight evolution, health status and quality of life during 2 years after bariatric surgery.

Materials and Methods: Retrospective cohort study carried out with individuals aged between 15 and 65 years old, who were submitted to any kind of bariatric surgery, from January 2010 to July 2011, in an Obesity Therapeutic Center. Anthropometric changes, biochemical and quality of life, coexisting conditions and the final result of the surgical intervention accessed by the Bariatric Analysis and Reporting Outcome System (BAROS) score were evaluated until 2 years after surgery.

Results: We evaluated 70 patients, $74.3 \%$ female, mean age $37.2 \pm 10.9$ years. The preoperative body mass index (BMI) was $45.5 \mathrm{~kg} / \mathrm{m}^{2}$. After 2 years of bariatric surgery, the average of weight loss was $37.3 \%$ (95\% Cl: 34.9 to $39.7 \%$ ). We observed $100 \%$ remission in cases of diabetes mellitus, hypertension and sleep apnea; $97.4 \%$ hypoventilation; $87.5 \%$ of venous stenosis; $75 \%$ of dyslipidemia and $61.7 \%$ of osteoarthritis. Regarding to the biochemical tests, we noted a reduction in total cholesterol and LDL in this period ( $p=0.042 ; p=0.021$, respectively). According to the BAROS specific questionnaire, the quality of life improved significantly in the first year after surgery, compared to the initial 6 months, in the areas of physical state, social life and capacity for working ( $p=0.008$; $p=0.001 ; p=0.035$; respectively), and in the second year in the sexual performance area $(p=0.008)$. In the final analysis of the surgical intervention the category "good" prevailed in all evaluated moments.

Conclusion: One year after the bariatric surgery, results showed weight reduction, improvement in cardiometabolic and general health, and quality of life of individuals.

Keywords: obesity; quality of life; bariatric surgery; state of health; Brazil.

\section{Correspondência:}

ELIANE ROSELI WINKELMANN

Departamento de Ciências da Vida - DCVida da UNIJUÍ

Rua do Comércio, no 3000 - Bairro Universitário

98700-000 ljuí, RS, Brasil

E-mail: elianew@unijui.edu.br 


\section{INTRODUÇÃO}

A obesidade tornou-se uma das principais preocupações de saúde entre adultos ${ }^{1,2}$ e adolescentes ${ }^{2}$ nos Estados Unidos. No Brasil, a Pesquisa Nacional de Saúde, realizada pelo Instituto Brasileiro de Geografia e Estatística em parceria com o Ministério da Saúde, mostrou que 56,9\% dos brasileiros estão acima do peso ${ }^{3}$. A abordagem tradicional de perda de peso que consiste em dieta, exercício e medicação atinge uma redução pouco significativa, em torno de $7-10 \%$ do peso corporal ${ }^{4,5}$. Além disso, a recuperação do peso prévio ou acima deste após a perda de peso ocorre em mais de $90 \%$ dos adultos ${ }^{6,7}$ e adolescentes ${ }^{8}$ submetidos à terapia nãocirúrgica.

A prevalência de obesidade extrema aumentou dramaticamente ao longo das últimas décadas, afetando quase $6 \%$ da população geral dos Estados Unidos ${ }^{9}$. Os procedimentos cirúrgicos bariátricos que restringem o tamanho do estômago ou levam à diminuição da absorção de nutrientes, são utilizados para tratar a obesidade grau 3 (Índice de Massa Corporal - IMC $\geq 40 \mathrm{~kg} / \mathrm{m}^{2}$ ). Estes procedimentos resultam em perda substancial de peso e podem ter efeito sobre comorbidades associadas a obesidade, tais como, a melhoria do controle glicêmico, ou mesmo reversão do diabetes mellitus tipo 2 e apnéia obstrutiva do sono ${ }^{10}$. No entanto, os procedimentos cirúrgicos bariátricos também carregam substanciais riscos, incluindo a morte.

A procura por tratamento de redução da obesidade tem-se intensificado nos últimos anos, assim como temse diversificado as técnicas e os métodos de tratamento ${ }^{11}$. A avaliação do tratamento cirúrgico tem exigido métodos específicos e adequados ${ }^{12,13}$. Como as intervenções cirúrgicas são cada vez mais intensas e aperfeiçoadas, o método Bariatric Analysis and Reporting Outcome System (BAROS) foi criado para avaliar a eficiência e comparar as técnicas cirúrgicas. Este método avalia as variações da qualidade de vida após a cirurgia bariátrica, a perda percentual do excesso de peso e a melhoria e/ou resolução das comorbidades ${ }^{14}$.

Sendo assim, o objetivo deste estudo foi descrever a evolução do peso, condições de saúde e qualidade de vida durante 2 anos após a cirurgia bariátrica.

\section{MATERIAIS E MÉTODOS}

Trata-se de uma coorte retrospectiva realizada com indivíduos, idade entre 15 a 65 anos, que realizaram qualquer tipo de procedimento cirúrgico bariátrico no período de Janeiro de 2010 a Julho de 2011 e que estavam em acompanhamento há, pelo menos, 2 anos em um Centro Terapêutico da Obesidade (CTO) do interior do Estado do Rio Grande do Sul. Os dados foram retirados do registro geral do indivíduo no CTO, os quais foram cadastrados pelos profissionais deste centro.

Foram excluídos os indivíduos que não realizaram o acompanhamento completo; as mulheres que engravidaram após a cirurgia (dentro do período de 2 anos) e os sujeitos que foram a óbito durante o período pesquisado. Após a aprovação da pesquisa pelo Comitê de Ética e Pesquisa da Universidade Regional do Noroeste do Estado do Rio Grande do Sul (UNIJUí), parecer no 272.017/2013, efetuou-se o levantamento dos dados. Foi resgatada toda a trajetória quanto à cirurgia bariátrica, desde o pré-operatório até o segundo ano após o procedimento, com dados coletados no período pré-operatório e aos 06, 12 e 24 meses pósoperatório.

Foram analisadas as seguintes variáveis: características demográficas; condições coexistentes associadas à obesidade e sua evolução após a intervenção cirúrgica; mudanças do peso corporal; exames bioquímicos e qualidade de vida, desde o pré-operatório até o segundo ano pós-operatório; bem como o resultado final da intervenção cirúrgica pela pontuação BAROS. Na rotina do serviço, o peso corporal em quilogramas $(\mathrm{kg})$ foi mensurado em uma balança de precisão (marca Cauduro, modelo CB para $300 \mathrm{~kg}$ ) e a altura aferida utilizando uma régua acessória, escalonada em centímetros, sendo os indivíduos orientados a permanecer com roupas leves e com os pés descalços ${ }^{15}$. Para determinar o IMC, utilizou-se o cálculo do peso corporal, em kg, dividido pelo quadrado da altura, em metros quadrados.

O método de bioimpedância elétrica (BIA) foi aplicado utilizando o aparelho marca Sanny modelo tetrapolar bio tetronic conforme recomendado ${ }^{16}$. As variáveis coletadas pelo BIA foram: o valor absoluto em $\mathrm{kg}$ e percentual de massa gorda e massa magra total.

Os dados bioquímicos dos níveis de colesterol total, lipoproteína de alta densidade (HDL), lipoproteína de baixa densidade (LDL), triglicerídeos e glicemia, foram obtidos dos prontuários dos indivíduos, uma vez que é rotina do serviço sua avaliação semestralmente, antes e após o procedimento cirúrgico bariátrico.

O protocolo BAROS foi aplicado pelos profissionais do CTO durante as revisões ambulatoriais dos indivíduos a partir do sexto mês pós-operatório. Os domínios avaliados nesse protocolo foram: perda de peso (percentual de perda do excesso de peso - \%PEP); avaliação clínica, realizada para identificação da melhora ou resolução das comorbidades como doença cardíaca, hipertensão arterial sistêmica (HAS), diabetes mellitus (DM), osteoartrite e apneia do sono; e a avaliação da qualidade de vida pelo questionário de Moorehead-Ardelt $I^{14,17}$. Cada domínio do questionário representa um valor numérico positivo 
ou negativo. O domínio avaliação da qualidade de vida contempla questões relacionadas à autoestima, estado físico, convívio/relacionamento social, capacidade de trabalho e desempenho sexual. A pontuação/classificação varia de $-1,00$ a 1,00 para cada questão, no qual $-1,00$ indica muito pior ou muito menos, $-0,50$ pior ou menos, 0 mesmo, 0,50 melhor ou mais e 1,00 muito melhor ou muito mais ${ }^{14,17}$.

A perda de peso foi avaliada pelo \%PEP onde se utilizou como parâmetro a tabela "padronizada de peso ideal", proposta pela Metropolitan Height and Weight Tables ${ }^{18}$. De acordo com os resultados, o \%PEP é classificada em cinco grupos e gera uma pontuação para o escore final de BAROS: ganho de peso (pontuação -1), perda de 0 a $24 \%$ (pontuação 0), perda de 25 a 49\% (pontuação 1), perda de 50 a $74 \%$ (pontuação 2) e perda de 75 a 100\% (pontuação 3) ${ }^{17}$.

Caso o indivíduo apresentasse alguma doença no préoperatório, incluindo HAS, DM tipo 2, doença cardiovascular, dislipidemias, apneia obstrutiva do sono, osteoartrite e infertilidade; este foi avaliado no pós-operatório para identificar se a comorbidade foi: agravada (pontuação -1); inalterada (pontuação 0); melhorada (pontuação 1); uma das maiores comorbidades foi resolvida e as outras foram melhoradas (pontuação 2); ou se todas as maiores comorbidades foram resolvidas e as outras foram melhoradas (pontuação 3). Os indivíduos que não apresentaram comorbidades no pré-operatório foram classificados como inalterados, recebendo pontuação igual a zero ${ }^{17}$.

As complicações do período pós-operatório foram classificadas como cirúrgicas e clínicas, maiores ou menores e precoces ou tardias ${ }^{17}$. Independente do número de complicações que tivessem ocorrido, foi deduzido da sua pontuação $-0,2$ pontos pelas complicações menores e menos um ponto pelas complicações maiores. Caso o indivíduo apresentasse uma complicação menor e uma maior, foi deduzido menos um ponto pelas complicações ${ }^{17}$. A pontuação zero foi atribuída nos casos em que foi realizada uma reoperação por causa da ocorrência de uma complicação. Os indivíduos que não apresentaram complicações receberam pontuação zero. Caso o indivíduo necessitasse de uma reoperação, foi reduzido um ponto do seu total de escores ${ }^{17}$.

A soma algébrica da pontuação de cada domínio resulta no escore final de BAROS que classifica o resultado da intervenção cirúrgica, como insuficiente (1 ou menos pontos), aceitável ( 1,1 a 3 pontos), bom ( 3,1 a 5 pontos), muito bom (5,1 a 7 pontos) e excelente (7,1 a 9 pontos $)^{17}$.

As medidas utilizadas nesta pesquisa foram a taxa de prevalência das comorbidades, calculada como a percentagem de indivíduos com a doença no período pré-operatório; e a taxa de remissão das comorbidades, calculada como a percentagem de indivíduos sem a doença nos períodos pós-operatórios, analisados entre aqueles que tinham a doença no período pré-operatório.

Todas as análises estatísticas foram realizadas utilizando o programa Statistical Package for the Social Science - SPSS (versão 18.0.0, Chicago, IL, EUA). A análise descritiva foi apresentada como média e intervalo de confiança de 95\%. Análise de medidas repetidas foi usada para avaliar alterações significativas do peso corporal, IMC e \% PEP. Análises de medidas repetidas com a covariável mudança de peso foram usadas para examinar se as alterações nas variáveis bioquímicas e qualidade de vida após o procedimento cirúrgico bariátrico foram explicadas pela mudança de peso corporal. Considerou-se significativo um $p<0,05$.

\section{RESULTADOS}

O número de indivíduos que realizaram o procedimento cirúrgico durante o período analisado foi de 133 sujeitos. Deste total, 54 foram excluídos por dados incompletos, 8 mulheres que engravidaram após a cirurgia e 1 indivíduo que foi a óbito. Portanto, o estudo envolveu um total de 70 indivíduos, destes $60(85,7 \%)$ realizaram o procedimento gastroplastia vertical com bypass gástrico em $\mathrm{Y}$ de Roux, sem anel e 10 (14,3\%) com anel.

$\mathrm{Na}$ avaliação pré-operatória, a média de idade foi de $37,2 \pm 10,9$ anos e a média do IMC foi de $45,5 \mathrm{~kg} / \mathrm{m}^{2}$ (variação, $33-87,8 \mathrm{~kg} / \mathrm{m}^{2}$ ); $65,7 \%$ dos indivíduos tinham um IMC maior que $40 \mathrm{~kg} / \mathrm{m}^{2}$; sendo a maioria do sexo feminino (74,3\%). Após 2 anos do procedimento cirúrgico, a média absoluta de redução de peso entre os indivíduos foi de 46,6 $\mathrm{kg}$ com leve aumento da altura. A média percentual de perda de peso foi de 37,3\% (IC95\%: 34,9-39,7\%), sendo 50,5\% (IC95\%: 56,2-70\%) de massa gorda e 24,4\% (IC95\%: 16,0-31,4\%) de massa magra (Tabela 1).

A DM tipo 2, HAS e apneia noturna estavam presentes em $25,7,47,1$ e 44,3\% dos indivíduos no pré-operatório, no segundo ano após a cirurgia bariátrica houve remissão de $100 \%$ dos casos. As maiores prevalências de remissão das condições coexistentes começam a partir do sexto mês pós-operatório estendendo-se até o primeiro ano pós procedimento. A incontinência urinária, condição presente em um único indivíduo no pré-operatório não teve resolução após a cirurgia (Tabela 2).

Com relação ao \%PEP, 100\% dos indivíduos submetidos ao procedimento bariátrico apresentaram uma perda entre 75 a 100\% do excesso de peso em curto prazo (até o primeiro mês após o procedimento). Já a longo prazo, 6 meses, 1 e 2 anos após o procedimento, prevaleceu o \%PEP entre 50 a 74\%, em 69,2, 93,1 e 84,6\% dos indivíduos, respectivamente. 
Tabela 1. Características demográficas e antropométricas dos indivíduos.

\begin{tabular}{|c|c|}
\hline Características & Total $(n=70)$ \\
\hline Idade - anos & $37,2 \pm 10,9$ \\
\hline \multicolumn{2}{|l|}{ Idade em quartis - n (\%) } \\
\hline $15-30$ anos & $17(24,3)$ \\
\hline $31-35$ anos & $18(25,7)$ \\
\hline $36-45$ anos & $18(25,7)$ \\
\hline $46-65$ anos & $17(24,3)$ \\
\hline \multicolumn{2}{|l|}{ Sexo - n. (\%) } \\
\hline Feminino & $52(74,3)$ \\
\hline Masculino & $18(25,7)$ \\
\hline \multicolumn{2}{|l|}{ Peso - Média (IC 95\%) } \\
\hline Pré-operatório - kg & $121,8(113,7-130,0)$ \\
\hline 2 anos - kg & $75,2(70,3-80,1)$ \\
\hline Mudança absoluta - kg & $-46,6(-51,7--41,6)$ \\
\hline Mudança em percentual & $-37,3(-39,7--34,9)$ \\
\hline \multicolumn{2}{|l|}{ Altura - Média (IC 95\%) } \\
\hline Pré-operatório - cm & $162,1(154,5-167,5)$ \\
\hline 2 anos $-\mathrm{cm}$ & $163,9(161,7-166,1)$ \\
\hline Mudança absoluta - cm & $1,8(1,4-7,0)$ \\
\hline Mudança em percentual & $1,2(1,1-4,1)$ \\
\hline \multicolumn{2}{|l|}{ IMC - Média (IC 95\%) } \\
\hline Pré-operatório - kg/m² & $45,5(42,7-48,2)$ \\
\hline 2 anos $-\mathrm{kg} / \mathrm{m}^{2}$ & $27,8(26,3-29,3)$ \\
\hline Mudança absoluta $-\mathrm{kg} / \mathrm{m}^{2}$ & $-17,7(-15,8--19,5)$ \\
\hline Mudança em percentual & $-38,9(-37,0--40,5)$ \\
\hline \multicolumn{2}{|c|}{ BIA massa magra - Média (IC 95\%) } \\
\hline Pré-operatório - kg & $65,6(59,4-71,7)$ \\
\hline 2 anos - kg & $49,6(36,9-62,2)$ \\
\hline Mudança absoluta - kg & $-16,0(-9,5--22,5)$ \\
\hline Mudança em percentual & $-24,4(-16,0--31,4)$ \\
\hline \multicolumn{2}{|c|}{ BIA massa gorda - Média (IC 95\%) } \\
\hline Pré-operatório - kg & $42,0(13,0-71,1)$ \\
\hline 2 anos $-\mathrm{kg}$ & $20,8(8,8-32,8)$ \\
\hline Mudança absoluta - kg & $-21,2(-7,3--49,8)$ \\
\hline Mudança em percentual & $-50,5(-56,2--70,0)$ \\
\hline
\end{tabular}

n: número; \%: percentual; IC: intervalo de confiança; IMC: índice de massa corporal; BIA: bioimpedância elétrica; kg: quilogramas; $\mathrm{cm}$ : centrímetros; $\mathrm{m}$ : metro.
Em todos os períodos do acompanhamento pós-operatório, o peso corporal e o IMC dos indivíduos estavam significativamente menores comparados à avaliação préoperatória, assim como, juntamente com o \%PEP em cada avaliação pós-operatória estavam significativamente inferiores a avaliação pós-operatória anterior, exceto para o peso e \%PEP entre o primeiro e segundo ano pós-operatório $(p=0,324 ; p=827$, respectivamente) (Tabela 3 ).

$\mathrm{Na}$ análise das variáveis bioquímicas com ajuste para a covariável mudança de peso corporal, verificou-se que a redução dos valores de colesterol total e LDL a longo prazo (segundo ano) comparada a avaliação préoperatória $(p=0,042 ; p=0,021$, respectivamente) foram explicadas pela mudança no peso corporal dos indivíduos (Tabela 3).

$\mathrm{Na}$ avaliação da qualidade de vida pós-operatória, segundo o questionário específico de BAROS, observouse uma melhora significativa dos domínios estado físico, convívio social e capacidade para o trabalho no primeiro ano após a cirurgia comparado ao sexto mês, $(p=0,008$; $p=0,001 ; p=0,035$; respectivamente), que se manteve até o segundo ano pós-operatório, exceto para a capacidade de trabalho $(p=0,525)$. No domínio desempenho sexual, houve uma melhora significativa somente no segundo ano após a cirurgia, comparado ao sexto mês pós-operatório $(p=0,008)$ (Tabela 4).

$\mathrm{Na}$ análise do resultado final da intervenção cirúrgica, segundo o protocolo BAROS, prevaleceu a categoria bom aos 6 meses (44,3\%), 1 ano (48,6\%) e 2 anos (51,4\%), seguido da categoria aceitável no sexto mês $(24,3 \%)$, da categoria muito bom no primeiro ano $(20,0 \%)$ e das categorias insuficiente e excelente $(17,2 \%)$ no segundo ano (Tabela 5).

Tabela 2. Prevalência pré-operatória e remissão pós-operatória de condições coexistentes.

\begin{tabular}{|c|c|c|c|c|c|c|c|c|c|c|}
\hline \multirow{4}{*}{ Condições } & \multirow{2}{*}{\multicolumn{2}{|c|}{ Pré-operatório }} & \multicolumn{8}{|c|}{ Pós-operatório } \\
\hline & & & PO imedi & & 60 mês & & 10 ano & & $2^{\circ}$ ano & \\
\hline & \multicolumn{2}{|c|}{ Prevalência da Condição } & \multicolumn{8}{|c|}{ Prevalência de Remissão da Condição } \\
\hline & $\begin{array}{c}\text { n de pacientes/ } \\
\text { n total }\end{array}$ & $\%$ & $\begin{array}{c}\text { n de pacientes/ } \\
\text { n total }\end{array}$ & $\%$ & $\begin{array}{l}\text { n de pacientes/ } \\
\text { n total }\end{array}$ & $\%$ & $\begin{array}{l}\text { n de pacientes/ } \\
\text { n total }\end{array}$ & $\%$ & $\begin{array}{l}\text { n de pacientes/ } \\
\text { n total }\end{array}$ & $\%$ \\
\hline Diabetes Mellitus & $18 / 70$ & 25,7 & $5 / 18$ & 27,8 & $17 / 18$ & 94,4 & $18 / 18$ & 100,0 & $18 / 18$ & 100,0 \\
\hline Hipertensão Arterial & $33 / 70$ & 47,1 & $13 / 33$ & 39,4 & $31 / 33$ & 94,0 & $33 / 33$ & 100,0 & $33 / 33$ & 100,0 \\
\hline Dislipidemia & $28 / 70$ & 40,0 & $0 / 28$ & 0,0 & $20 / 28$ & 71,4 & $21 / 28$ & 75,0 & $21 / 28$ & 75,0 \\
\hline Apneia noturna & $31 / 70$ & 44,3 & $0 / 31$ & 0,0 & $31 / 31$ & 100,0 & $31 / 31$ & 100,0 & $31 / 31$ & 100,0 \\
\hline Hipoventilação & $39 / 70$ & 55,7 & $4 / 39$ & 10,2 & $36 / 39$ & 92,3 & $38 / 39$ & 97,4 & $38 / 39$ & 97,4 \\
\hline Osteoartrite & $47 / 70$ & 67,1 & $3 / 47$ & 6,4 & $18 / 47$ & 38,3 & $28 / 47$ & 59,6 & $29 / 47$ & 61,7 \\
\hline Estase venosa & $8 / 70$ & 11,4 & $0 / 8$ & 0,0 & $3 / 8$ & 37,5 & $7 / 8$ & 87,5 & $7 / 8$ & 87,5 \\
\hline Incontinência urinária & $1 / 70$ & 1,4 & $0 / 1$ & 0,0 & $0 / 1$ & 0,0 & $0 / 1$ & 0,0 & $0 / 1$ & 0,0 \\
\hline
\end{tabular}

PO: pós-operatório; n: número; \%: percentual. 
Tabela 3. Dados antropométricos e variáveis bioquímicas pré procedimento bariátrico e a curto e longo prazo pós cirurgia bariátrica.

\begin{tabular}{|c|c|c|c|c|c|c|}
\hline \multirow{2}{*}{ Variáveis } & \multirow{2}{*}{ Pré-operatório } & \multicolumn{4}{|c|}{ Pós-operatório } & \multirow{2}{*}{$\mathbf{p}$} \\
\hline & & PO imediato & $6^{0}$ mês & 10 ano & $2 \mathbf{o}$ ano & \\
\hline \multicolumn{7}{|c|}{ Dados antropométricos } \\
\hline Peso - kg & $121,8(113,7-130,0)^{\#}$ & $110,4(101,4-119,4)^{* \#}$ & $88,6(81,6-95,6)^{* \#}$ & $78,5(72,8-84,3)^{* \#}$ & $75,2(70,3-80,1)^{* \#}$ & $<0,001$ \\
\hline $\mathrm{IMC}-\mathrm{kg} / \mathrm{m}^{2}$ & $45,5(42,7-48,2 \#$ & $41,2(38,0-44,4)^{* \#}$ & $33,0(30,6-35,4)^{* \#}$ & $29,3(27,3-31,3)^{* \#}$ & $27,8(26,3-29,3) * \#$ & $<0,001$ \\
\hline$\%$ PEP & - & $89,2(87,7-90,7)^{\#}$ & $71,7(70,0-73,4)^{\#}$ & $64,0(61,9-66,0)^{\#}$ & $62,7(60,2-65,3)^{\#}$ & $<0,001 \mid$ \\
\hline \multicolumn{7}{|l|}{ Exames bioquímicos } \\
\hline Colesterol total & $194,0(180,3-207,6)$ & $160,9(151,0-170,8)$ & $161,7(149,0-174,3)$ & $154,0(129,5-178,5)$ & $153,0(137,4-168,6)^{*}$ & $0,03 \|$ \\
\hline HDL & $51,0(47,0-55,1)$ & $44,8(39,8-49,8)$ & $51,2(47,4-55,0)$ & $57,1(46,6-67,7)$ & $59,4(46,6-72,3)$ & $0,316^{\|}$ \\
\hline LDL & $110,8(98,6-123,0)$ & $89,4(79,3-99,4)$ & $87,8(76,8-98,8)$ & $79,2(59,7-98,7)$ & $68,5(53,9-83,0)^{*}$ & $0,02^{\prime \prime}$ \\
\hline Triglicerídeos & $163,9(125,0-202,8)$ & $109,2(92,2-126,1)$ & $115,0(97,7-132,3)$ & $103,7(56,7-151,0)$ & $88,6(71,6-105,7)$ & $0,266^{\prime \prime}$ \\
\hline Glicemia & $93,8(80,0-107,4)$ & $91,4(79,0-103,7)$ & $88,1(78,7-97,6)$ & $78,3(72,5-84,1)$ & $81,6(76,5-86,7)$ & $0,533^{\prime \prime}$ \\
\hline
\end{tabular}

PO: pós-operatório; IMC: índice de massa corporal; kg: quilograma; m: metro; \%PEP: percentual de perda de excesso de peso;

HDL: lipoproteína de alta densidade; LDL: lipoproteína de baixa densidade.

análise de medidas repetidas; II análises de medidas repetidas com a covariável mudança de peso; * diferença significativa versus pré-operatório;

\# diferença significativa versus qualquer período pós-operatório.

Tabela 4. Qualidade de vida após o procedimento bariátrico.

\begin{tabular}{lcccc}
\hline \multicolumn{1}{c}{ Domínios } & \multicolumn{3}{c}{ Pós-operatório } & p \\
\cline { 2 - 3 } Autoestima & $\mathbf{6} \mathbf{o}$ mês & $\mathbf{1}^{\mathbf{o}}$ ano & $\mathbf{2}$ ano & \\
Estado físico & $0,821 \pm 0,256$ & $0,900 \pm 0,202$ & $0,918 \pm 0,187$ & 0,240 \\
Convívio social & $0,325 \pm 0,156$ & $0,385 \pm 0,154^{*}$ & $0,389 \pm 0,141^{*}$ & 0,031 \\
Capacidade de trabalho & $0,307 \pm 0,196$ & $0,408 \pm 0,137^{*}$ & $0,410 \pm 0,177^{*}$ & 0,025 \\
Desempenho sexual & $0,311 \pm 0,223$ & $0,389 \pm 0,177^{*}$ & $0,377 \pm 0,202$ & 0,026 \\
\hline
\end{tabular}

Análises de medidas repetidas com a covariável mudança de peso.

* Diferença significativa versus $6^{\circ}$ mês.

Tabela 5. Resultado final de BAROS a curto e longo prazo no pós-operatório.

\begin{tabular}{|c|c|c|c|}
\hline \multirow{2}{*}{$\begin{array}{l}\text { Resultado final } \\
\text { de BAROS }\end{array}$} & \multicolumn{3}{|c|}{ Pós-operatório } \\
\hline & 6o mês & 10 ano & 20 ano \\
\hline Insuficiente & $3(4,3)$ & $2(2,8)$ & $12(17,2)$ \\
\hline Aceitável & $17(24,3)$ & $10(14,3)$ & $8(11,4)$ \\
\hline Bom & $31(44,3)$ & $34(48,6)$ & $36(51,4)$ \\
\hline Muito bom & $10(14,3)$ & $14(20,0)$ & $2(2,8)$ \\
\hline Excelente & $9(12,8)$ & $10(14,3)$ & $12(17,2)$ \\
\hline
\end{tabular}

\section{DISCUSSÃO}

A maioria dos indivíduos deste estudo obteve uma melhora significativa do peso corporal, estado de saúde e qualidade de vida. Não houve indivíduo cuja condição tenha sido agravada ou que tenha permanecido inalterada. Os resultados da cirurgia bariátrica com avaliações sistemáticas em curtos períodos de tempo para além do primeiro ano após o procedimento cirúrgico tem sido raramente descritos ${ }^{19-21}$.
Considerando as dificuldades habitualmente encontradas na perda ponderal por meio das medidas convencionais (terapia não cirúrgica), a cirurgia bariátrica apresenta-se na atualidade como um método muito eficiente para a indução de perda ponderal importante e sustentada em obesos mórbidos $^{10,22}$. A perda do excesso de peso é considerada um dos principais parâmetros para definir o sucesso da cirurgia bariátrica, já que, após o emagrecimento, ocorre comprovada melhora das comorbidades ${ }^{23}$. Nos dados apresentados, o percentual médio de perda de excesso de peso no primeiro e segundo ano de acompanhamento está dentro do esperado, $60 \%$ e $65 \%$ respectivamente, de acordo com avaliação proposta por Shi et al. ${ }^{24}$. É ainda, melhor do que os resultados publicados por Bobowicz et al. ${ }^{19}, 43,6 \%$ no primeiro ano e $46,6 \%$ no segundo ano pós cirurgia, e pouco inferiores aos encontrados por Mingrone et al. ${ }^{20}$, os quais evidenciaram, no segundo ano de seguimento, $69,4 \%$ e $68,1 \%$ de perda de excesso de peso em indivíduos submetidos a derivação biliopancreática e bypass gástrico, respectivamente. No presente estudo não foi realizada análise por tipo de procedimento cirúrgico bariátrico. 
A redução média absoluta do peso corporal no primeiro e segundo ano de seguimento, encontrada neste estudo, foi semelhante ao relatado em estudo longitudinal retrospectivo ${ }^{25}$ e prospectivo ${ }^{20}$. No estudo de Pedrosa et al..$^{25}$, o qual avaliou retrospectivamente 205 pacientes antes e após cirurgia bariátrica pela técnica Fobi-Capela (Bypass Gástrico em Y de Roux), encontrou uma perda de peso de 43,0 kg no primeiro ano e de $47,5 \mathrm{~kg}$ no segundo ano pós operatório. No estudo de Mingrone et al. ${ }^{20}$, o qual avaliou prospectivamente 18, 19 e 19 pacientes submetidos a terapia médica, derivação biliopancreática e bypass gástrico, respectivamente, encontrou, ao final do segundo ano de seguimento uma perda de peso de $8,3 \mathrm{~kg}$ no grupo terapia médica, 48,3 kg no grupo submetido a derivação biliopancreática e $45,5 \mathrm{~kg}$ no grupo submetido a bypass gástrico.

As mulheres constituem a população que mais procura a cirurgia bariátrica. A maior prevalência do sexo feminino entre os indivíduos do presente estudo foi semelhante à relatada em estudos brasileiros prévios ${ }^{25,26}$ e também em estudos internacionais ${ }^{19,21}$.

Apesar do estudo analisar uma população adulta jovem, o excesso de peso e suas comorbidades podem amplificar os fatores de risco cardiovasculares, entre eles a DM tipo 2, a HAS e a dislipidemia. Entre adultos que se submetem a bypass gástrico, a remissão de diabetes tipo 2 ocorre em 50 a $70 \%$ dos casos, e a remissão de pressão arterial elevada ocorre em 40\% ${ }^{27,28}$. Em um estudo randomizado e controlado, cujo objetivo era comparar a eficácia de dois tipos de cirurgia bariátrica (bypass gástrico e derivação biliopancreática) com a terapia médica convencional em pacientes com obesidade mórbida e diabetes tipo 2, foi encontrado, aos 2 anos de seguimento pós-operatório, que nenhuma remissão de diabetes ocorreu no grupo tratamento convencional, contra $75 \%$ no grupo bypass gástrico e $95 \%$ no grupo derivação biliopancreátrica ${ }^{20}$. No presente estudo, observou-se uma taxa de remissão de diabetes e hipertensão arterial em 100\% dos indivíduos que tinham diabetes tipo 2 e hipertensão arterial na linha de base, um achado superior ao constatado em estudos anteriores $20,27,28$. Esses resultados, juntamente com os resultados da alta taxa de remissão de dislipidemia e melhora nos níveis de colesterol total e LDL, leva a sugerir que indivíduos submetidos à cirurgia bariátrica precocemente associada a uma boa adesão ao tratamento e a mudanças no estilo de vida podem ter um potencial superior para inversão das consequências cardiometabólicas da obesidade. Houve mudanças importantes no estilo de vida desses indivíduos, o que certamente contribui e influencia nos resultados obtidos neste estudo, os quais, não podem ser atribuídos exclusivamente ao procedimento cirúrgico, visto que no segundo ano após a cirurgia, 86,5\% dos indivíduos estavam realizando exercício físico regularmente. Além disso, do período pré-operatório para o segundo ano após a cirurgia, houve abolição do uso excessivo de bebidas alcoólicas (2,9\%) e redução da taxa de tabagismo (de 10\% para 1,4\%), ingesta excessiva de sal (de 44,3 para 1,4\%) e de gordura (de 38,6 para 1,4\%).

As dislipidemias mostram-se altamente prevalentes neste estudo, caracterizando um risco cardiovascular aumentado precoce nesses indivíduos. Diferentemente do constatado com relação às taxas de remissão de diabetes, às taxas de remissão da dislipidemia foram menores no presente estudo comparado ao estudo de Mingrone et al. ${ }^{20}$, visto que nesse estudo $100 \%$ dos pacientes submetidos a cirurgia tiveram seus níveis de lipídeos normalizados após 2 anos do procedimento cirúrgico, contra $75 \%$ no presente estudo.

A apneia do sono é altamente prevalente e debilitante em adultos com obesidade mórbida ${ }^{29}$. Em estudos mais antigos, a apneia do sono melhorou consideravelmente, mas resolveu em apenas metade dos $\operatorname{casos}^{30,31}$, já em estudo mais recente resolveu em $92 \%$ e melhorou em $8 \%{ }^{32}$. A probabilidade de resolução da apneia do sono após redução do peso pode ser maior em pessoas com apneia menos severa no préoperatório $^{31}$, como os dados deste estudo sugerem. Nguyen et al..$^{33}$ relatam que a perda de peso após a cirurgia bariátrica melhora a mecânica pulmonar, como resultado há o aumento da complacência pulmonar, reduzindo a obstrução das vias aéreas ${ }^{34}$. Neste estudo, observou-se uma resolução de quase todos os casos de hipoventilação pulmonar no segundo ano pós-operatório.

Tem sido demonstrado que a qualidade de vida póscirurgia bariátrica melhora significativamente ${ }^{19,22,26,35}$. A melhoria da qualidade de vida, em curto prazo, ocorre pela mudança da imagem corporal após a cirurgia bariátrica ${ }^{35}$. No estudo de Hemiö et al..$^{36}$ as pontuações do questionário de qualidade de vida foram significativamente melhores em todos os domínios depois de 1 ano a partir da cirurgia e essa melhora foi mantida em 5 anos de acompanhamento, no entanto, a pontuação não atinge o nível da população em geral. Outro estudo $^{37}$ de coorte longitudinal prospectivo com maior tempo de seguimento, realizado em hospitais universitários e centros de obesidade da Alemanha, que envolveu 152 indivíduos submetidos a tratamento cirúrgico, encontrou melhorias na qualidade de vida física em todas as avaliações pós operatórias. Embora os indivíduos tenham obtido melhorias iniciais em domínios relacionados à depressão, ansiedade, autoestima e aspectos mentais da qualidade de vida, essa melhoria se deteriorou na avaliação aos 9 anos e foi comparável ou pior que os níveis pré cirúrgicos. No presente estudo, os indivíduos apresentaram melhora em todos os domínios relacionados à qualidade de vida, principalmente no primeiro ano pós- 
operatório, período a mais longo prazo, em que a maior parte do excesso de peso foi perdida. Em análise dos prontuários, todos responderam que sua condição clínica foi melhorada ou resolvida, ratificando que a perda de peso ocasiona a resolução das comorbidades ${ }^{10}$, proporcionando melhora da qualidade de vida ${ }^{17}$, entretanto, devemos considerar que o tempo de levantamento deste estudo foi inferior aos supracitados.

A principal limitação deste estudo foi a sua natureza retrospectiva, entretanto, a robustez do banco de dados, associado com o tempo do estudo e modelo longitudinal podem diminuir essa limitação. Os pontos fortes do presente estudo incluem a análise de dados de indivíduos de locais geograficamente distintos, pois o CTO estudado é um centro de referência para a região, e a realização de avaliações sistemáticas em curtos períodos de tempo foi importante para analisar especificamente em que período as melhorias ocorrem e se deterioram.

Em conclusão, após um ano da realização da cirurgia bariátrica, observa-se redução do peso, melhora na saúde cardiometabólica e geral e na qualidade de vida dos indivíduos. Foi documentado a durabilidade clinicamente significativa da perda de peso e melhorias chave no estado de saúde e qualidade de vida durante 2 anos após cirurgia bariátrica em indivíduos de 15 a 65 anos de idade. A perda de peso pós procedimento foi significativa durante os 2 anos de acompanhamento em comparação com a sua avaliação pré-operatória. Estudos para avaliar a durabilidade da perda de peso durante prazos maiores, potenciais melhorias com relação às condições coexistentes, e o risco de ocorrência de efeitos adversos, bem como o custo envolvido, podem proporcionar uma melhor compreensão do papel da cirurgia bariátrica no tratamento da obesidade extrema.

\section{REFERÊNCIAS}

1. Mokdad AH, Marks JS, Stroup DF, Gerberding JL. Actual causes of death in the United States, 2000. JAMA. 2004;291(10):1238-45. http://dx.doi.org/10.1001/jama.291.10.1238

2. Ogden CL, Carroll MD, Kit BK, Flegal KM. Prevalence of childhood and adult obesity in the United States, 2011-2012. JAMA. 2014;311(8):806-14. http://dx.doi.org/10.1001/jama.2014.732

3. Instituto Brasileiro de Geografia e Estatística. Pesquisa Nacional de Saúde 2013 [Internet]. Rio de Janeiro: IBGE; 2013 [cited 2016 Jan 20]. Available from: http://www.ibge.gov.br

4. Wadden TA, Webb VL, Moran CH, Bailer BA. Lifestyle Modification for Obesity. Circulation. 2012;125(9):1157-70. http://dx.doi. org/10.1161/CIRCULATIONAHA.111.039453

5. Moyer VA, U. S. Preventive Services Task Force. Screening for and Management of Obesity in Adults: U.S. Preventive Services Task Force Recommendation Statement. Ann Intern Med. 2012;157(5):373-9. http://dx.doi.org/10.7326/0003-4819-157-5-201209040-00475
6. Safer DJ. Diet, behavior modification, and exercise: a review of obesity treatments from a long-term perspective. South Med J. 1991;84(12):1470-4. http://dx.doi.org/10.1097/00007611199112000-00015

7. Wadden TA, Sternberg JA, Letizia KA, Stunkard AJ, Foster GD. Treatment of obesity by very low calorie diet, behavior therapy, and their combination: a five-year perspective. Int J Obes. 1989;13 Suppl 2:39-46.

8. Levine MD, Ringham RM, Kalarchian MA, Wisniewski L, Marcus MD. Is family-based behavioral weight control appropriate for severe pediatric obesity? Int J Eat Disord. 2001;30(3):318-28. http://dx.doi.org/10.1002/eat.1091

9. Flegal KM, Carroll MD, Kit BK, Ogden CL. Prevalence of obesity and trends in the distribution of body mass index among US adults, 1999-2010. JAMA. 2012;307(5):491-7. http://dx.doi.org/10.1001/ jama.2012.39

10. Buchwald H, Avidor Y, Braunwald E, Jensen MD, Pories W, Fahrbach K, Schoelles K. Bariatric surgery: a systematic review and metaanalysis. JAMA. 2004;292(14):1724-37. http://dx.doi.org/10.1001/ jama.292.14.1724

11. Segal A, Fandi-o J. Indicações e contra indicações para realização das Operações Bariátricas. Rev Bras Psiquiatr. 2002;24(Supl 3):6872. http://dx.doi.org/10.1590/S1516-44462002000700015

12. Pinto S, Bosi ML. Muito mais do que pensam: percepções e experiências acerca da obesidade. Physis. 2010;20(2):443-57.

13. Victorzon M, Tolonen P. Bariatric Analysis and Reporting Outcome System (BAROS) following laparoscopic adjustable gastric banding in Finland. Obes Surg. 2001;11(6):740-3. http://dx.doi. org/10.1381/09608920160558696

14. Oria H, Moorehead M. Bariatric analysis and reporting outcome system (BAROS). Obes Surg. 1998. http://dx.doi. org/10.1381/096089298765554043

15. Heyward VH, Stolarczyk LM. Avaliação da composição corporal aplicada. Phorte Editora: São Paulo; 2000.

16. Slinde F, Rossander-Hulthen L. Bioelectrical impedance: effect of three identical meals on diurnal impedance variation and calculation of body composition. Am J Clin Nutr. 2001;74:474-8.

17. Oria HE, Moorehead MK. Updated bariatric analysis and reporting outcome system (BAROS). Surg Obes Relat Dis. 2009;5(1):60-6. http://dx.doi.org/10.1016/j.soard.2008.10.004

18. Metropolitan Life Foundation. Metropolitan height and weight tables. Stat Bull. 1983;64:2-9.

19. Bobowicz $M$, Lehmann A, Orlowski $M$, Lech $P$, Michalik $M$. Preliminary outcomes 1 year after laparoscopic sleeve gastrectomy based on Bariatric Analysis and Reporting Outcome System (BAROS), Obes Surg. 2011;21(12):1843-8. http://dx.doi.org/10.1007/s11695011-0403-4

20. Mingrone G, Panunzi S, Gaetano AD, Guidone C, laconelli A, Leccesi L, Nanni G, Pomp A, Castagneto M, Ghirlanda G, Rubino F. Bariatric surgery versus conventional medical therapy for type 2 diabetes. N Engl J Med. 2012;366(17):1577-85. http://dx.doi. org/10.1056/NEJMoa1200111

21. Still CD, Wood C, Chu X, Manney C, Strodel W, Petrick A, Gabrielsen J, Mirshahi T, Argyropoulos G, Seiler J, Yung M, Benotti P, Gerhard 
GS. Clinical factors associated with weight loss outcomes after Rouxen-Y gastric bypass surgery. Obesity (Silver Spring). 2014;22(3): 888-94. http://dx.doi.org/10.1002/oby.20529

22. van der Beek ES, Geenen R, de Heer FA, van der Molen AB, van Ramshorst B. Quality of life long-term after body contouring surgery following bariatric surgery: sustained improvement after 7 years. Plast Reconstr Surg. 2012;130(5):1133-9. http://dx.doi. org/10.1097/PRS.0b013e318267d51d

23. Gloy VL, Briel M, Bhatt DL, Kashyap SR, Schauer PR, Mingrone G, Bucher HC, Nordmann AJ. Bariatric surgery versus non-surgical treatment for obesity: a systematic review and meta-analysis of randomised controlled trials. BMJ. 2013;347:f5934. http://dx.doi. org/10.1136/bmj.f5934

24. Shi X, Karmali S, Sharma AM, Birch DW. A review of laparoscopic sleeve gastrectomy for morbid obesity. Obes Surg. 2010;20(8):11717. http://dx.doi.org/10.1007/s11695-010-0145-8

25. Pedrosa IV, Burgos MGPA, Souza NC, Morais CN. Aspectos nutricionais em obesos antes e após a cirurgia bariátrica. Rev Col Bras Cir. 2009;36(4):316-22. http://dx.doi.org/10.1590/S010069912009000400008

26. Khawali C, Ferraz MB, Zanella MT, Ferreira SRG. Avaliação da qualidade de vida de pacientes com obesidade grave submetidos à cirurgia bariátrica em um sistema público de saúde. Arq Bras Endocrinol Metab. 2012;56(1):33-8. http://dx.doi.org/10.1590/ S0004-27302012000100006

27. Schauer PR, Bhatt DL, Kirwan JP, Wolski K, Brethauer SA, Navaneethan SD, Aminian A, Pothier CE, Kim ESH, Nissen SE, Kashyap SR; STAMPEDE Investigators. Bariatric surgery versus intensive medical therapy for diabetes 3-year outcomes. N Engl J Med. 2014;370(21):2002-13. http://dx.doi.org/10.1056/ NEJMoa1401329

28. Courcoulas AP, Christian NJ, Belle SH, Berk PD, Flum DR, Garcia L, Horlick M, Kalarchian MA, King WC, Mitchell JE, Patterson EJ, Pender JR, Pomp A, Pories WJ, Thirlby RC, Yanovski SZ, Wolfe BM; Longitudinal Assessment of Bariatric Surgery (LABS) Consortium. Weight change and health outcomes at 3 years after bariatric surgery among individuals with severe obesity. JAMA. 2013;310(22):241625. http://dx.doi.org/10.1001/jama.2013.280928
29. Valencia-Flores $M$, Orea $A$, Castano VA, Resendiz $M$, Rosales M, Rebollar V, Santiago V, Gallegos J, Campos RM, González J, Oseguera J, García-Ramos G, Bliwise DL. Prevalence of sleep apnea and electrocardiographic disturbances in morbidly obese patients. Obes Res. 2000;8(3):262-9. http://dx.doi.org/10.1038/oby.2000.31

30. Charuzi I, Lavie P, Peiser J, Peled R. Bariatric surgery in morbidly obese sleep-apnea patients: short- and long-term follow-up. Am J Clin Nutr. 1992;55(2 Suppl):594-6.

31. Valencia-Flores M, Orea A, Herrera M, Santiago V, Rebollar V, Castano VA, Oseguera J, Pedroza J, Sumano J, Resendiz M, GarcíaRamos G. Effect of bariatric surgery on obstructive sleep apnea and hypopnea syndrome, electrocardiogram, and pulmonary arterial pressure. Obes Surg. 2004;14(6):755-62. http://dx.doi. org/10.1381/0960892041590773

32. Steffen R, Potoczna N, Bieri N, Fritz H. Successful multi-intervention treatment of severe obesity: a 7 -year prospective study with $96 \%$ follow-up. Obes Surg. 2009;19(1):3-12. http://dx.doi.org/10.1007/ s11695-008-9675-8

33. Nguyen NT, Hinojosa MW, Smith BR, Gray J, Varela E. Improvement of restrictive and obstructive pulmonary mechanics following laparoscopic bariatric surgery. Surgery Endoscopic. 2009;23(4):80812. http://dx.doi.org/10.1007/s00464-008-0084-9

34. Ceneviva R, Silva GA, Viegas MM, Sankarankutt AK, Chueire FB. Cirurgia bariátrica e apnéia do sono. Medicina. 2006;39(2):235-45. http://dx.doi.org/10.11606/issn.2176-7262.v39i2p236-245

35. Stunkard AJ, Stinnett JL, Smoller JW. Psychological and social aspects of the surgical treatment of obesity. Am J Psychiatry. 1986;143(4):417-29. http://dx.doi.org/10.1176/ajp.143.4.417

36. Helmiö M, Salminen P, Sintonen H, Ovaska J, Victorzon M. A 5-year prospective quality of life analysis following laparoscopic adjustable gastric banding for morbid obesity. Obes Surg. 2011;21(10):158591. http://dx.doi.org/10.1007/s11695-011-0425-y

37. Herpertz S, Müller A, Burgmer R, Crosby RD, Zwaan M, Legenbauer T. Health-related quality of life and psychological functioning 9 years after restrictive surgical treatment for obesity. Surg Obes Relat Dis. 2015;11(6):1361-70. http://dx.doi.org/10.1016/j. soard.2015.04.008 